\title{
Global Koppelman Formulas on (Singular) Projective Varieties
}

\section{Mats Andersson ${ }^{1}$}

Received: 11 May 2017 / Published online: 27 August 2018

(c) The Author(s) 2018

\begin{abstract}
Let $i: X \rightarrow \mathbb{P}^{N}$ be a projective manifold of dimension $n$ embedded in projective space $\mathbb{P}^{N}$, and let $L$ be the pullback to $X$ of the line bundle $\mathcal{O}_{\mathbb{P}^{N}}(1)$. We construct global explicit Koppelman formulas on $X$ for smooth $(0, *)$-forms with values in $L^{s}$ for any $s$. The same construction works for singular, even non-reduced, $X$ of pure dimension, if the sheaves of smooth forms are replaced by suitable sheaves $\mathcal{A}_{X}^{*}$ of $(0, *)$-currents

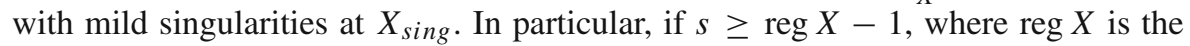
Castelnuovo-Mumford regularity, we get an explicit representation of the well-known vanishing of $H^{0, q}\left(X, L^{s-q}\right), q \geq 1$. Also some other applications are indicated.
\end{abstract}

Keywords Projective variety $\cdot$ Integral formula $\cdot$ dbar-equation

Mathematics Subject Classification 32A26 · 32C30 - 32J99

\section{Introduction}

During the last decade, global Koppelman formulas for $\bar{\partial}$ on various special projective varieties have been constructed, see, e.g., [17-19,22-24]. The aim of this paper is to present a quite general explicit construction of intrinsic ${ }^{1}$ Koppelman formulas on any projective, possibly non-reduced, subvariety $i: X \rightarrow \mathbb{P}^{N}$ of pure dimension $n$.

Let us first assume that $X$ is smooth; even in this case, such global formulas are previously known only in case $X$ is (locally) a complete intersection in $\mathbb{P}^{N}$. Let $L \rightarrow X$ be the restriction of the ample line bundle $\mathcal{O}_{\mathbb{P}^{N}}(1)$ to $X$, and let $\mathcal{E}_{X}^{0, q}\left(L^{r}\right)$ denote the

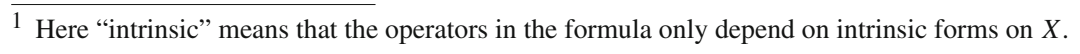

The author was partially supported by the Swedish Research Council.

$\bowtie \quad$ Mats Andersson

matsa@math.chalmers.se

1 Department of Mathematics, Chalmers University of Technology and the University of Gothenburg, 41296 Gothenburg, Sweden 
sheaf of smooth $(0, q)$-forms on $X$ with values in $L^{r}$. We introduce integral operators $\mathcal{K}: \mathcal{E}^{0, q+1}\left(X, L^{s}\right) \rightarrow \mathcal{E}^{0, q}\left(X_{\text {reg }}, L^{s}\right)$ and $\mathcal{P}: \mathcal{E}^{0, q}\left(X, L^{s}\right) \rightarrow \mathcal{E}^{0, q}\left(X, L^{s}\right)$ such that the Koppelman formula

$$
\phi(z)=\bar{\partial} \mathcal{K} \phi+\mathcal{K}(\bar{\partial} \phi)+\mathcal{P} \phi
$$

holds on $X$. In some situations, see below, we can choose the operators so that $\mathcal{P} \phi=0$; if $\bar{\partial} \phi=0$, then $\psi=\mathcal{K} \phi$ is a smooth solution to $\bar{\partial} \psi=\phi$ on $X$. In certain cases, we can choose $\mathcal{P}$ such that $\mathcal{P}: \mathcal{E}^{0,0}\left(X, L^{s}\right) \rightarrow \mathcal{O}\left(\mathbb{P}^{N}, \mathcal{O}(s)\right)$. Then $\mathcal{P} \phi$ is a holomorphic extension to $\mathbb{P}^{N}$ of a holomorphic section $\phi$ of $L^{r}$ on $X$. We get no new existence results; the novelty is that we have explicit formulas for the global solutions and for the holomorphic extensions. The operators $\mathcal{K}$ are given by kernels $k(\zeta, z)$ that are defined on $X \times X$ and integrable in $\zeta$ for any $z \in X$. Simply speaking the operators locally behave like standard integral operators for $\bar{\partial}$ in $\mathbb{C}^{n}$; in particular, they extend to $L^{p}$-spaces, etc., and all classical local norm estimates hold.

Let us now turn our attention to the case when $i: X \rightarrow \mathbb{P}^{N}$ is a subvariety of pure dimension $n$. In case $X$ is reduced, there are well-known definitions of smooth forms and currents on $X$, cf. Sect. 3. In [9] are introduced reasonable definitions of sheaves $\mathcal{E}_{X}^{0, *}$ of smooth $(0, *)$-forms and suitable sheaves of currents even in the non-reduced case, see Sect. 6 below. In [6] and [9] are introduced, by means of local Koppelman formulas, fine sheaves $\mathcal{A}_{X}^{k}$ (in fact, modules over $\mathcal{E}_{X}^{0, *}$ ) of $(0, q)$-currents on $X$, or any pure-dimensional analytic space, with the following properties: There are sheaf inclusions $\mathcal{E}_{X}^{0, q} \subset \mathcal{A}_{X}^{q}$ with equality on $X_{\text {reg }}$, whereas $\mathcal{A}_{X}^{q}$ have "mild" singularities at $X_{\text {sing }}$, and

$$
0 \rightarrow \mathcal{O} \rightarrow \mathcal{A}_{X}^{0} \stackrel{\bar{\partial}}{\rightarrow} \mathcal{A}_{X}^{1} \stackrel{\bar{\partial}}{\rightarrow} \cdots
$$

is a (fine) resolution of the structure sheaf $\mathcal{O}_{X}$ of holomorphic functions on $X$. By the abstract de Rham theorem, we therefore have canonical isomorphisms

$$
H^{k}\left(X, L^{s}\right) \simeq \frac{\operatorname{Ker}\left(\mathcal{A}^{k}\left(X, L^{s}\right) \stackrel{\bar{\partial}}{\rightarrow} \mathcal{A}^{k+1}\left(X, L^{s}\right)\right)}{\left.\operatorname{Im}\left(\mathcal{A}^{k-1}\left(X, L^{s}\right)\right) \stackrel{\bar{\partial}}{\rightarrow} \mathcal{A}^{k}\left(X, L^{s}\right)\right)}, \quad k \geq 1
$$

In this paper, we construct integral operators

$$
\mathcal{K}: \mathcal{A}^{q+1}\left(X, L^{s}\right) \rightarrow \mathcal{A}^{q}\left(X, L^{s}\right), \quad \mathcal{P}: \mathcal{A}^{q}\left(X, L^{s}\right) \rightarrow \mathcal{E}^{0, q}\left(X, L^{s}\right)
$$

such that again the Koppelman formula (1.1) holds on $X$. In the reduced case, the operators $\mathcal{K}$ are given by kernels $k(\zeta, z)$ that are defined on $X_{\text {reg }} \times X$, locally integrable in $\zeta$ for $z \in X_{\text {reg }}, \mathcal{P}$ are given by kernels $p(\zeta, z)$ that are smooth on $X_{\text {reg }} \times X$, and the integrals

$$
\mathcal{K} \phi(z)=\int_{X} k(\zeta, z) \wedge \phi(\zeta), z \in X_{r e g}, \quad \mathcal{P} \phi(z)=\int_{X} p(\zeta, z) \wedge \phi(\zeta), z \in X,
$$

exist as principal values at $X_{\text {sing }}$. For the non-reduced case, see Sect. 6 . As in the smooth case, in good situations $\mathcal{P} \phi$ vanishes so we get explicit solutions to $\bar{\partial} \psi=\phi$, and extensions of holomorphic sections from $X$ to $\mathbb{P}^{N}$. 
Remark 1 It was proved already in [21] that if $X$ is a local reduced complete intersection and $\phi$ is a smooth $\bar{\partial}$-closed form, then there is locally a smooth solution to $\bar{\partial} \psi=\phi$ on $X_{r e g}$. The case with a general $X$ was proved only in [5]. The analogous result for a local non-reduced space is a special case of the main result in [9]. It is known that in general there is no solution that is smooth across $X_{\text {sing }}$, see, e.g., [6, Example 1.1].

Let $J_{X}$ be the homogeneous ideal in the graded ring $S=\mathbb{C}\left[z_{0}, \ldots, z_{N}\right]$ associated with $X$. Let $S(-r)$ be the module $S$ but with the grading shifted by $r$. There is a free graded resolution

$$
0 \rightarrow M_{N_{0}} \stackrel{a_{N_{0}}}{\longrightarrow} \cdots \stackrel{a_{3}}{\longrightarrow} M_{2} \stackrel{a_{2}}{\longrightarrow} M_{1} \stackrel{a_{1}}{\longrightarrow} M_{0}
$$

of the homogeneous module $M_{J}:=S / J_{X}$; i.e.,

$$
M_{0}=S, \quad M_{k}=S\left(-d_{k}^{1}\right) \oplus \cdots \oplus S\left(-d_{k}^{r_{k}}\right)
$$

$a_{k}=\left(a_{k}^{i j}\right)$ are matrices of homogeneous forms with

$$
\operatorname{deg} a_{k}^{i j}=d_{k}^{j}-d_{k-1}^{i}
$$

(1.4) is exact, and the cokernel of the right-most mapping is precisely $S / J_{X}$. Since 0 is not an associated prime ideal of $J_{X}$ it follows from [11, Corollary 20.14] that one can choose (1.4) such that $N_{0} \leq N$. Our integral formulas are explicitly constructed out of a resolution (1.4).

Recall that the (Castelnuovo-Mumford) regularity of $X$ is defined as the regularity of the ideal $J_{X}$ which turns out to be 1 plus the regularity of the module $S / J_{X}$, so that

$$
\operatorname{reg} X=\max _{k, i}\left(d_{k}^{i}-k\right)+1
$$

if (1.4) is a minimal free resolution of $S / J_{X}$, cf. [12, Ch. 4]. It is well known, see, e.g., [12, Proposition 4.16], that

$$
H^{q}\left(X, L^{s-q}\right)=0, s \geq \operatorname{reg} X-1, q \geq 1,
$$

and that the natural mapping

$$
\mathcal{O}\left(\mathbb{P}^{N}, \mathcal{O}(s)\right) \rightarrow \mathcal{O}\left(X, L^{s}\right)
$$

is surjective for $s \geq \operatorname{reg} X-1$.

In [4, Example 3.4] is described an extension operator that provides an explicit proof of surjectivity of (1.7), in case $X$ is reduced. The non-reduced case is obtained in precisely the same way following the ideas in [9]. By appropriate choices of operators $\mathcal{K}$ we can give an explicit proof of the vanishing of (1.6), provided that $X$ is irreducible. That is, we have 
Theorem 1.1 Let $X$ be a, possibly non-reduced, irreducible, subvariety of $\mathbb{P}^{N}$ of pure dimension $n$ and assume that $s \geq \operatorname{reg} X-1$. For each $q \geq 1$ there is an integral operator $\mathcal{K}: \mathcal{A}^{q}\left(X, L^{s-q}\right) \rightarrow \mathcal{A}^{q-1}\left(X, L^{s-q}\right)$ such that $\bar{\partial} \mathcal{K} \phi=\phi$ if $\phi \in \mathcal{A}^{q}\left(X, L^{s-q}\right)$ and $\bar{\partial} \phi=0$.

In fact, for fixed $q$ it is enough that $s \geq \max _{\ell \leq N-q} d_{\ell}^{i}-(N-q)$; this follows from the proof below. When $X$ is not irreducible a slightly less sharp version of the theorem still holds, see Proposition 7.2.

Koppelman formulas on $\mathbb{P}^{n}$ were found by Götmark, [17], and on more general symmetric spaces in [18]. In [20], explicit formulas for the $\bar{\partial}$-equation are used on a smooth Riemann surface embedded in $\mathbb{P}^{2}$, for $L^{1}$-estimates. Similar formulas were also introduced in [19], cf. Sect. 8.2 below. Koppelman formulas for global, even nonreduced, complete intersections are constructed in the recent papers [23,24], cf. Sect. 8 below.

As already mentioned, the main novelty in this paper is Koppelman formulas for an arbitrary embedded projective variety $X$. We think that these formulas will be of interest even when $X$ is smooth. We prove Theorem 1.1 as an illustration of the utility and indicate some other applications in Sect.7.1. We hope that our Koppelman formulas will be useful for other purposes as well.

In Sect. 2 we describe, based on $[3,4,17]$, how one can obtain weighted integral formulas on $\mathbb{P}^{N}$. We need some elements from residue theory that we have collected in Sect.3. In Sects. 4 and 5, we then describe the construction of our Koppelman formulas on a pure-dimensional subvariety. In order to keep the technicalities on a reasonable level, we restrict here to the case where $X$ is reduced. The reader who is mainly interested in the smooth case can just think that $X_{\text {sing }}$ is empty, in that way avoiding a lot of technicalities. In Sect. 6, we discuss the non-reduced case.

Remark 2 The local study of the $\bar{\partial}$-equation on non-smooth spaces by $L^{2}$-methods was initiated by Pardon and Stern, [31-33], and has been developed by a number of authors since then, notably Fornaess, Gavosto, Ovrelid, Ruppenthal, Vassiliadou, see., e.g., [14-16,28-30,34,35].

The equation has also been studied by local and semi-global integral formulas in, e.g., $[5,6,21,25,26,37]$.

\section{Integral Representation on $\mathbb{P}^{N}$}

We first describe how one can generate weighted Koppelman formulas on $\mathbb{P}^{N}$ for sections of a holomorphic vector bundle $F \rightarrow \mathbb{P}^{N}$. This is an adaption of an idea from [1] to $\mathbb{P}^{N}$, following [3,4]; see also [17].

Let $\pi: \mathbb{C}_{z}^{N+1} \backslash\{0\} \rightarrow \mathbb{P}_{z}^{N}$ be the natural projection and let $\mathcal{U} \subset \mathbb{P}^{N}$ be an open set. Recall that a form $\xi$ in $\pi^{-1} \mathcal{U} \subset \mathbb{C}_{z}^{N+1} \backslash\{0\}$ is projective, i.e., the pullback of a form $\xi$ in $\mathcal{U}$, if and only if $\xi$ is homogeneous and $\delta_{z} \xi=\delta_{\bar{z}} \xi=0$, where $\delta_{z}$ and $\delta_{\bar{z}}$ are interior multiplication by $\sum_{0}^{N} z_{j}\left(\partial / \partial z_{j}\right)$ and its conjugate, respectively. We will identify forms in $\mathcal{U}$ by projective forms in $\pi^{-1} \mathcal{U}$.

Let $\mathcal{O}_{z}(k)$ denote the pullback of $\mathcal{O}(k) \rightarrow \mathbb{P}_{z}^{N}$ to $\mathbb{P}_{\zeta}^{N} \times \mathbb{P}_{z}^{N}$ under the projection $\mathbb{P}_{\zeta}^{N} \times \mathbb{P}_{z}^{N} \rightarrow \mathbb{P}_{z}^{N}$ and define $\mathcal{O}_{\zeta}(k)$ analogously. 
Throughout this paper we will only consider forms and currents that only contain holomorphic differentials with respect to $\zeta$, whereas anti-holomorphic differentials with respect to both $z$ and $\zeta$ may occur.

Notice that

$$
\eta=2 \pi i \sum_{0}^{N} z_{i} \frac{\partial}{\partial \zeta_{i}}
$$

is a section of $\mathcal{O}_{z}(1) \otimes \mathcal{O}_{\zeta}(-1) \otimes T_{1,0}\left(\mathbb{P}_{\zeta}^{N}\right)$ on $\mathbb{P}_{\zeta}^{N} \times \mathbb{P}_{z}^{N}$. Contraction $\delta_{\eta}$ by $\eta$ defines a mapping

$$
\delta_{\eta}: \mathcal{C}^{\ell+1, q}\left(\mathcal{O}_{\zeta}(k) \otimes \mathcal{O}_{z}(j)\right) \rightarrow \mathcal{C}^{\ell, q}\left(\mathcal{O}_{\zeta}(k-1) \otimes \mathcal{O}_{z}(j+1)\right),
$$

where $\mathcal{C}^{\ell, q}\left(\mathcal{O}_{\zeta}(k) \otimes \mathcal{O}_{z}(j)\right)$ denotes the sheaf of currents of bidegree $(\ell, q)$ that take values in $\mathcal{O}_{\zeta}(k) \otimes \mathcal{O}_{z}(j)$. Notice that $\delta_{\eta}$ only affects holomorphic differentials with respect to $\zeta$. Given a vector bundle $L \rightarrow \mathbb{P}_{\zeta}^{n} \times \mathbb{P}_{z}^{n}$, let

$$
\mathcal{L}^{v}(L)=\bigoplus_{j} \mathcal{C}^{j, j+v}\left(\mathcal{O}_{\zeta}(j) \otimes \mathcal{O}_{z}(-j) \otimes L\right)
$$

If

$$
\nabla_{\eta}=\delta_{\eta}-\bar{\partial}
$$

then $\nabla_{\eta}: \mathcal{L}^{v}(L) \rightarrow \mathcal{L}^{v+1}(L)$ and $\nabla_{\eta}^{2}=0$. Furthermore, if $L^{\prime}$ is a line bundle and $\phi, \psi$ are sections of $\mathcal{L}^{v}(L)$ and $\mathcal{L}^{\nu^{\prime}}\left(L^{\prime}\right)$, respectively, then $\phi \wedge \psi$ is a section of $\mathcal{L}^{v+v^{\prime}}\left(L \otimes L^{\prime}\right)$, and

$$
\nabla_{\eta}(\phi \wedge \psi)=\nabla_{\eta} \phi \wedge \psi+(-1)^{\operatorname{deg} \phi} \phi \wedge \nabla_{\eta} \psi
$$

Notice that

$$
b=\frac{|\zeta|^{2} \bar{z} \cdot d \zeta-(\bar{z} \cdot \zeta) \bar{\zeta} \cdot d \zeta}{|\zeta|^{2}|z|^{2}-|\bar{\zeta} \cdot z|^{2}}
$$

is a (1,0)-form on $\mathbb{P}_{\zeta}^{N} \times \mathbb{P}_{z}^{N} \backslash \Delta$ with values in $\mathcal{O}_{\zeta}(1) \otimes \mathcal{O}_{z}(-1)$ such that $\delta_{\eta} b=1$; here $\Delta$ is the diagonal in $\mathbb{P}_{\zeta}^{N} \times \mathbb{P}_{z}^{N}$.

Lemma 2.1 The form

$$
B=\frac{b}{\nabla_{\eta} b}=\frac{b}{1-\bar{\partial} b}=b+b \wedge \bar{\partial} b+\cdots+b \wedge(\bar{\partial} b)^{N-1}
$$

is an integrable section of $\mathcal{L}^{-1}$ and

$$
\nabla_{\eta} B=1-[\Delta]_{d \zeta}
$$


where the last term is the component of the current of integration $[\Delta]$ that has full degree $N$ in $d \zeta$.

Proof Let us consider the affinization where $\zeta_{0} \neq 0$. In the affine coordinates $\zeta_{j}^{\prime}=$ $\zeta_{j} / \zeta_{0}, j=1, \ldots, N$, and the frame $z_{0} / \zeta_{0}$ for $\mathcal{O}_{z}(1) \otimes \mathcal{O}_{\zeta}(-1)$, we have

$$
\eta=2 \pi i \sum_{1}^{N}\left(\zeta_{j}^{\prime}-z_{j}^{\prime}\right) \frac{\partial}{\partial \zeta_{j}^{\prime}}
$$

It is readily checked that $|b| \leq C\left|\zeta^{\prime}-z^{\prime}\right|$ and $\left|\delta_{\eta} b\right| \geq C\left|\zeta^{\prime}-z^{\prime}\right|^{2}$, and therefore (2.1) follows, cf. [1, Example 4].

Given a vector bundle $F \rightarrow \mathbb{P}^{N}$, let $F_{z}$ denote the pullback of $F$ to $\mathbb{P}_{\zeta}^{N} \times \mathbb{P}_{z}^{N}$ under the natural projection $\mathbb{P}_{\zeta}^{N} \times \mathbb{P}_{z}^{N} \rightarrow \mathbb{P}_{z}^{N}$ and define $F_{\zeta}$ analogously. A weight with respect to $F$ is a smooth section $g$ of $\mathcal{L}^{0}\left(\operatorname{Hom}\left(F_{\zeta}, F_{z}\right)\right)$ such that $\nabla_{\eta} g=0$ and $g_{0}=I_{F}$ on the diagonal in $\mathbb{P}_{\zeta}^{N} \times \mathbb{P}_{z}^{N}$, where $g_{0}$ denotes the term in $g$ with bidegree $(0,0)$. In general, we let lower index on a form denote degree with respect to holomorphic differentials of $\zeta$. Notice that if $g$ is a weight with respect to $F$, then from (2.1) we get

$$
\nabla_{\eta}(g \wedge B)=\left(g-[\Delta]_{d \zeta}\right) I_{F} .
$$

Identifying terms of full degree in $d \zeta$ thus

$$
\bar{\partial}(g \wedge B)_{N}=\left([\Delta]_{d \zeta}-g_{N}\right) I_{F} .
$$

By Stokes' theorem we get the following Koppelman formula, cf. [17] and [18].

Proposition 2.2 Let $g$ be a weight with respect to $F \rightarrow \mathbb{P}^{N}$. Then for $\phi \in$ $\mathcal{E}^{0, q}\left(\mathbb{P}^{N}, F \otimes \mathcal{O}(-N)\right)$ we have

$$
\phi(z)=\bar{\partial}_{z} \int_{\zeta}(g \wedge B)_{N} \wedge \phi+\int_{\zeta}(g \wedge B)_{N} \wedge \bar{\partial} \phi+\int_{\zeta} g_{N} \wedge \phi .
$$

Example 1 It is easy to check that

$$
\alpha=\alpha_{0,0}+\alpha_{1,1}=\frac{z \cdot \bar{\zeta}}{|\zeta|^{2}}-\bar{\partial} \frac{\bar{\zeta} \cdot d \zeta}{2 \pi i|\zeta|^{2}}
$$

is a projective form in $\left.\mathcal{L}^{0}\left(\mathcal{O}_{\zeta}(-1) \oplus \mathcal{O}_{z}(1)\right)\right)$ such that

$$
\nabla_{\eta} \alpha=0
$$

Since $\alpha_{0}$ is equal to $I_{\mathcal{O}(1)}$ on the diagonal, $\alpha$ is a weight with respect to $F=\mathcal{O}(1)$. For each natural number $\rho$ therefore $g=\alpha^{\rho}$ is a weight with respect to $F=\mathcal{O}(\rho)$. For $\ell \geq-N$, we thus have the Koppelman formula

$$
\phi(z)=\bar{\partial}_{z} \int_{\zeta}\left(\alpha^{\ell+N} \wedge B\right)_{N} \wedge \phi+\int_{\zeta}\left(\alpha^{\ell+N} \wedge B\right)_{N} \wedge \bar{\partial} \phi+\int_{\zeta}\left(\alpha^{N+\ell}\right)_{N} \wedge \phi .
$$


Since $\alpha$ is holomorphic in $z$ and has no differentials $d \bar{z}$, the last term in (2.5) vanishes if $q \geq 1$, and for degree reasons also if $q=0$ and $\ell \leq-1$.

We thus have an explicit proof of the well-known vanishing $H^{0, q}\left(\mathbb{P}^{N}, \mathcal{O}(\ell)\right)=0$ for $\ell \geq-N$ if $1 \leq q \leq N$, and for $q=0$ if $\ell \leq-1$.

Remark 3 Using the transposed integral operators in Example 1, we get an explicit proof of the vanishing $H^{N, q}\left(\mathbb{P}^{N}, \mathcal{O}(\ell)\right)=0$ for $\ell \leq N, 0 \leq 1 \leq N-1$. Since $K_{\mathbb{P}^{N}}=\mathcal{O}(-N-1)$ we therefore get that $H^{0, q}\left(\mathbb{P}^{N}, \mathcal{O}(\ell)\right)=0$ for $\ell \leq-1,0 \leq q \leq$ $N-1$.

Let us now consider a weight with respect to $\mathcal{O}(-1)$. Let

$$
\tau=\frac{d \bar{z} \cdot d \zeta}{2 \pi i|z|^{2}}=\frac{\sum_{0}^{N} d \bar{z}_{j} \wedge d \zeta_{j}}{2 \pi i|z|^{2}}
$$

Then

$$
\beta=2 \pi i\left[\delta_{\zeta} \frac{\delta_{\bar{z}} \tau}{1-\tau}\right]=2 \pi i\left[\frac{\delta_{\zeta} \delta_{\bar{z}} \tau}{1-\tau}+\frac{\delta_{\zeta} \tau \wedge \delta_{\bar{z}} \tau}{(1-\tau)^{2}}\right]
$$

is a projective form. More explicitly,

$$
\beta=\frac{\bar{z} \cdot \zeta}{|z|^{2}}+\frac{\bar{z} \cdot \zeta}{|z|^{2}} \frac{d \bar{z} \cdot d \zeta}{2 \pi i|z|^{2}}+\frac{\bar{z} \cdot d \zeta \wedge \zeta \cdot d \bar{z}}{2 \pi i|z|^{4}}+\cdots
$$

so each term has the same degree in $d \zeta$ as in $d \bar{z}$, and is holomorphic in $\zeta$.

Proposition 2.3 The form $\beta$ is a weight with respect to $\mathcal{O}(-1)$.

Proof Clearly, cf. (2.6), $\beta_{0,0}=I_{\mathcal{O}(-1)}$ on the diagonal. We claim that

$$
\nabla_{\eta} \frac{\delta_{\bar{z}} \tau}{1-\tau}=1
$$

Since $\delta_{\zeta}$ anti-commutes with $\nabla_{\eta}$ and $\delta_{\zeta} 1=0$ the proposition follows. To see (2.7), notice that

$$
\delta_{\eta} \tau=-\frac{z \cdot d \bar{z}}{|z|^{2}}, \quad \bar{\partial} \tau=\delta_{\eta} \tau \wedge \tau, \quad \delta_{\eta} \delta_{\bar{z}} \tau=1,
$$

so that

$$
\bar{\partial} \delta_{\bar{z}} \tau=\tau+\delta_{\eta} \tau \wedge \delta_{\bar{z}} \tau=\tau+\gamma
$$

Therefore,

$$
\nabla_{\eta} \delta_{\bar{z}} \tau=1-\tau-\gamma
$$

and

$$
\nabla_{\eta} \tau=\delta_{\eta} \tau \wedge(1-\tau)
$$


so that

$$
\nabla_{\eta} \tau \wedge \delta_{\bar{z}} \tau=(1-\tau) \wedge \gamma
$$

It follows that

$$
\nabla_{\eta} \frac{\delta_{\bar{z}} \tau}{1-\tau}=\frac{1-\tau-\gamma}{1-\tau}+\frac{\nabla_{\eta} \tau \wedge \delta_{\bar{z}} \tau}{(1-\tau)^{2}}=1
$$

in view of (2.8), (2.9), and (2.10).

Thus for $(0, q)$-forms $\phi$ with values in $\mathcal{O}(\ell), \ell \leq-N$, we get from Proposition 2.2 the Koppelman formula

$$
\phi(z)=\bar{\partial} \int_{\zeta}\left(B \wedge \beta^{-N-\ell}\right)_{N} \wedge \phi+\int_{\zeta}\left(B \wedge \beta^{-N-\ell}\right)_{N} \wedge \bar{\partial} \phi+\int_{\zeta}\left(\beta^{-N-\ell}\right)_{N} \wedge \phi
$$

For degree reasons the last term vanishes if $0 \leq q \leq N-1$, so we get back the wellknown vanishing $H^{0, q}\left(\mathbb{P}^{N}, \mathcal{O}(\ell)\right)=0$ for $\ell \leq-N$. In case $q=N$, the obstruction term vanishes when $\ell=-N$, and when $\ell \leq-N-1$ it vanishes if and only if

$$
\int \psi \wedge \phi=0
$$

for each holomorphic $(N, 0)$-form with values in $\mathcal{O}(-\ell)$. That is, $\bar{\partial} v=\phi$ is solvable if and only if (2.11) holds. Of course this is precisely what we get by considering the transposed operators with the weight $\alpha$, cf. Remark 3. However, in the non-smooth case, we have no obvious canonical bundle so we cannot consider transposed operators in the same simple way; therefore this weight $\beta$ will play a role.

For future reference we prove

Proposition 2.4 The forms

$$
\gamma_{j}=\delta_{\zeta}\left[\frac{\delta_{\bar{z}} \tau}{1-\tau} \wedge d \zeta_{j}\right]
$$

are projective and

$$
\nabla_{\eta} \gamma_{j}=\beta z_{j}-\zeta_{j}
$$

Proof Clearly $\delta_{\zeta} \gamma_{j}=0=\delta_{\bar{z}} \gamma_{j}$ and thus $\gamma_{j}$ is a projective form. By (2.7) we have that

$$
\nabla_{\eta}\left[\frac{\delta_{\bar{z}} \tau}{1-\tau} \wedge d \zeta_{j}\right]=d \zeta_{j}-2 \pi i \frac{\delta_{\bar{z}} \tau}{1-\tau} z_{j}
$$

Since $\nabla_{\eta}$ and $\delta_{\zeta}$ anti-commute, the proposition follows. 


\section{Some Preliminaries}

Let $X$ be any reduced analytic space of pure dimension $n$. By definition there is, locally, some embedding $i: X \rightarrow \Omega \subset \mathbb{C}^{N}$. Let $\mathcal{J}_{X} \subset \mathcal{O}_{\Omega}$ be the ideal sheaf of holomorphic functions in $\Omega$ that vanish on $X$. Then the sheaf of holomorphic functions on $X$, the structure sheaf $\mathcal{O}_{X}$, is represented as $\mathcal{O}_{X}=\mathcal{O}_{\Omega} / \mathcal{J}_{X}$. If $\Phi$ is a smooth form in $\Omega$ we say that $\Phi$ is in $\mathcal{K} e r i^{*}$ if $i^{*} \Phi$ vanishes on $X_{\text {reg }}$. We define the sheaf

$$
\mathcal{E}_{X}^{p, *}=\mathcal{E}_{\Omega}^{p, *} / \mathcal{K} e r i^{*}
$$

of smooth forms on $X$, and have a natural mapping $i^{*}: \mathcal{E}_{\Omega}^{p, *} \rightarrow \mathcal{E}_{X}^{p, *}$. One can prove that $\mathcal{E}_{X}^{p, *}$ so defined is independent of the choice of embedding and is thus an intrinsic sheaf on $X$. We define the sheaf $\mathcal{C}_{X}^{p \text {,* }}$ of currents as the dual of $\mathcal{E}_{X}^{n-p, n-*}$. More concretely this means that currents $\tau$ in $\mathcal{C}_{X}^{p \text {,* }}$ are identified with currents $i_{*} \tau$ in $\mathcal{C}_{\Omega}^{p+N-n, *+N-n}$ such that $i_{*} \tau$ vanish on $\mathcal{K} e r i^{*}$ so that $\tau . i^{*} \Phi=i_{*} \tau$. $\Phi$ for test forms $\Phi$. Clearly $\bar{\partial}$ is defined on smooth forms and extends to currents by duality. Also the wedge product $\phi \wedge \tau$ is well defined as long as at least one of the factors is smooth. Thus the currents form a module over the smooth forms.

We say that a current in $\mathbb{C}_{S}^{M}$ of the form

$$
\frac{\gamma}{s_{1}^{a_{1}} \cdots s_{r}^{a_{r}}} \bar{\partial} \frac{1}{s_{r+1}^{a_{r+1}}} \wedge \cdots \bar{\partial} \frac{1}{s_{r^{\prime}}^{a_{r^{\prime}}}},
$$

where $\gamma$ is a test form, is elementary. A current $\tau$ on $X$ is pseudomeromorphic if locally it is a finite sum of direct images under holomorphic mappings of elementary currents; see, e.g., [10] for a precise definition and basic properties. The pseudomeromorphic currents form a sheaf $\mathcal{P} \mathcal{M}_{X}$ that is closed under multiplication by $\mathcal{E}_{X}^{p, *}$ and the action of $\bar{\partial}$. Given a pseudomeromorphic current $\tau$ in an open set $\mathcal{U}$ and a subvariety $V \subset \mathcal{U}$, the natural restriction of $\tau$ to $\mathcal{U} \backslash V$ has a canonical extension to a pseudomeromorphic current $\mathbf{1}_{\mathcal{U} \backslash V} \tau$ such that

$$
\mathbf{1}_{V} \tau:=\tau-\mathbf{1}_{\mathcal{U} \backslash V} \tau
$$

has support on $V$. If $\xi$ is a smooth form, then

$$
\xi \wedge \mathbf{1}_{V} \tau=\mathbf{1}_{V} \xi \wedge \tau
$$

Let $\chi$ be a smooth function on $[0, \infty)$ that is 0 in a neighborhood of 0 and 1 in a neighborhood of $\infty$ and let $h$ be a tuple of holomorphic functions, or a section of some holomorphic Hermitian vector bundle such that the zero set of $h$ is precisely $V$. Then

$$
\mathbf{1}_{\mathcal{U} \backslash V} \tau=\lim _{\delta \rightarrow 0} \chi(|h| / \delta) \tau
$$

We say that a current $a$ in $X$ is almost semi-meromorphic, $a \in A S M(X)$, if there is a smooth modification $\pi: X^{\prime} \rightarrow X$, a generically non-vanishing holomorphic section $\sigma$ of a line bundle $L \rightarrow X^{\prime}$ and a smooth $L$-valued form $\gamma$ such that 


$$
a=\pi_{*}(\gamma / \sigma) .
$$

Let $Z S S(a)$ be the smallest analytic subset of $X$ such that $a$ is smooth in $X \backslash Z S S(a)$. It follows that $Z S S(a)$ has positive codimension. Clearly an almost semi-meromorphic $a$ is pseudomeromorphic.

Proposition 3.1 [10, Theorem 4.8] Given any pseudomeromorphic $\tau$ and $a \in$ $A S M(X)$ the current a $\wedge \tau$ a priori defined in $X \backslash Z S S(a)$ has a unique pseudomeromorphic extension to a pseudomeromorphic current in $X$, also denoted $a \wedge \tau$, such that $\mathbf{1}_{Z S S(a)} a \wedge \tau=0$.

Pseudomeromorphic currents have some important geometric properties, see, e.g., [10]:

Proposition 3.2 Assume that the pseudomeromorphic current $\tau$ has support on a germ of an analytic variety $V$.

(i) If the holomorphic function $h$ vanishes on $V$, then $\bar{h} \tau=0$ and $d \bar{h} \wedge \tau=0$.

(ii) If $\tau$ has bidegree $(*, p)$ and $V$ has codimension $\geq p+1$, then $\tau=0$.

We refer to (ii) as the dimension principle.

\section{A Structure Form Associated to $X$}

Let $i: X \rightarrow \mathbb{P}^{N}$ be a reduced subvariety of pure dimension $n$, and let (1.4) be a free graded resolution of the $S$-module $M_{X}=S / J_{X}$. In particular, then $a_{1}=$ $\left(a_{1}^{11}, \ldots, a_{1}^{1 r_{1}}\right)$ is a tuple of homogeneous forms that define the homogeneous ideal $J_{X}$ in the graded ring $S=\mathbb{C}\left[z_{0}, \ldots, z_{N}\right]$. Let $E_{k}^{j}$ be disjoint trivial line bundles over $\mathbb{P}^{N}$ with basis elements $e_{k, j}$ and let

$$
E_{k}=\left(E_{k}^{1} \otimes \mathcal{O}\left(-d_{k}^{1}\right)\right) \oplus \cdots \oplus\left(E_{k}^{r_{k}} \otimes \mathcal{O}\left(-d_{k}^{r_{k}}\right)\right), \quad E_{0} \simeq \mathbb{C} .
$$

Then

$$
0 \rightarrow E_{N_{0}} \stackrel{a_{N_{0}}}{\longrightarrow} \cdots \stackrel{a_{3}}{\longrightarrow} E_{2} \stackrel{a_{2}}{\longrightarrow} E_{1} \stackrel{a_{1}}{\longrightarrow} E_{0} \rightarrow 0
$$

is a complex of vector bundles over $\mathbb{P}^{N}$ that is pointwise exact outside $Z$, and the corresponding complex of locally free sheaves

$$
0 \rightarrow \mathcal{O}\left(E_{N_{0}}\right) \stackrel{a_{N_{0}}}{\longrightarrow} \cdots \stackrel{a_{3}}{\longrightarrow} \mathcal{O}\left(E_{2}\right) \stackrel{a_{2}}{\longrightarrow} \mathcal{O}\left(E_{1}\right) \stackrel{a_{1}}{\longrightarrow} \mathcal{O}\left(E_{0}\right)
$$

over $\mathbb{P}^{N}$ is a resolution of the sheaf $\mathcal{O}\left(E_{0}\right) / \mathcal{J}_{X}$, where $\mathcal{J}_{X} \subset \mathcal{O}_{\mathbb{P}^{N}}$ is the ideal sheaf associated with $X$. See, e.g., [7, Sect. 6]. We equip $E_{k}$ with the natural Hermitian metric

$$
|\xi(z)|_{E_{k}}^{2}=\sum_{j=1}^{r_{k}}\left|\xi_{j}(z)\right|^{2}|z|^{2 d_{k}^{j}}
$$


if $\xi=\left(\xi_{1}, \ldots, \xi_{r_{k}}\right)$, so that (4.1) becomes a Hermitian complex. In [7] were introduced pseudomeromorphic currents

$$
U=U_{1}+\cdots+U_{N_{0}}, \quad R=R_{1}+\cdots+R_{N_{0}}
$$

on $\mathbb{P}^{N}$ associated to (4.1) with the following properties: The currents $U_{k}$ are almost semi-meromorphic $(0, k-1)$-currents, smooth outside $X$, that take values in $\operatorname{Hom}\left(E_{0}, E_{k}\right) \simeq E_{k}$, and $R_{k}$ are $(0, k)$-currents with support on $X$, taking values in $\operatorname{Hom}\left(E_{0}, E_{k}\right) \simeq E_{k}$. Moreover, we have the relations

$$
a_{1} U_{1}=I_{E_{0}}, \quad a_{k+1} U_{k+1}-\bar{\partial} U_{k}=-R_{k}, \quad k \geq 1,
$$

which can be compactly written as

$$
\nabla_{a} U=I_{E_{0}}-R
$$

if

$$
\nabla_{a}=a-\bar{\partial}=a_{1}+a_{2}+\cdots a_{N_{0}}-\bar{\partial} .
$$

If $\Phi$ is a section of $\mathcal{O}=\mathcal{O}\left(E_{0}\right)$, then the current $R \Phi$ vanishes if and only if $\Phi$ is in $\mathcal{J}_{X}$, see [7, Theorem 1.1].

Let $X_{k}$ be the analytic subset of $\mathbb{P}^{N}$ where $a_{k}$ does not have optimal rank. Then

$$
\ldots X_{k+1} \subset X_{k} \ldots X_{N-n+1} \subset X_{\text {sing }} \subset X=X_{N-n}=\cdots=X_{1} \text {. }
$$

Since $\mathcal{J}_{X}$ has pure dimension

$$
\operatorname{codim} X_{k} \geq k+1, \quad k \geq N-n+1
$$

see [11, Corollary 20.14].

By the dimension principle $R_{k}=0$ for $k<N-n$. Moreover, there are almost semimeromorphic $\operatorname{Hom}\left(E_{k}, E_{k+1}\right)$-valued $(0,1)$-currents $\alpha_{k+1}$, smooth outside $X_{k+1}$, such that

$$
R_{k+1}=\alpha_{k+1} R_{k}
$$

there. By (4.6) and the dimension principle it follows that this equality must hold across $X_{k+1}$ if the right-hand side is interpreted in the sense of Proposition 3.1. By a simple induction argument, using (4.6) and the dimension principle, it follows that

$$
\mathbf{1}_{X_{\text {sing }} R} R=0 .
$$

Lemma 4.1 If $\Phi$ is a smooth $(0, *)$-form, then $i^{*} \Phi=0$ on $X_{\text {reg }}$ if and only if $\Phi R=0$. It follows that $R \phi$ is well-defined for $\phi$ in $\mathcal{E}_{X}^{0, *}$. 
Proof Locally at a point $x \in X_{\text {reg }}$ we can choose coordinates $(z, w)$ such that $X=$ $\{w=0\}$. By a Taylor expansion of $\Phi$ in $w$, using that $w_{j} R=\bar{w}_{j} R=d \bar{w}_{j} \wedge R=0$, cf. Proposition 3.2 (i), we find that that $R \Phi=0$ if and only if $i^{*} \Phi=0$. If $\Phi R=0$ on $X_{\text {reg }}$ it follows from (3.1) and (4.7) that $\Phi R=0$ identically.

Let $\Omega=\delta_{z}\left(d z_{0} \wedge \cdots \wedge d z_{N}\right)$ be the unique, up to a multiplicative constant, nonvanishing global $(N, 0)$-form with values in $\mathcal{O}(N+1)$. From [6, Proposition 3.3] we have

Proposition 4.2 There is a unique almost semi-meromorphic current $\omega=\omega_{0}+\cdots+\omega_{n}$ on $X$ that is smooth on $X_{\text {reg }}$, $\omega_{\ell}$ have bidegree $(n, \ell)$ and take values in $E^{\ell}:=$ $i^{*} E_{N-n+\ell}$, and

$$
i_{*} \omega=\Omega \wedge R, \quad i_{*} \omega_{\ell}=\Omega \wedge R_{N-n+\ell} .
$$

We say that $\omega$ is a structure form on $X$.

For any smooth form $\xi$ on $\mathbb{P}^{N}$ there is a unique form $\vartheta(\xi)$ such that

$$
\vartheta(\xi) \wedge \Omega=\xi_{N},
$$

where $\xi_{N}$ denotes the components of $\xi$ of bidegree $(N, *)$. From (4.8) and (4.9) we have that

$$
\xi_{N} \wedge R=\vartheta(\xi) \wedge \Omega \wedge R=i_{*}(\vartheta(\xi) \wedge \omega),
$$

where we in the last term, for simplicity, write $\vartheta(\xi) \wedge \omega$ rather then $i^{*} \vartheta(\xi) \wedge \omega$.

Lemma 4.3 Let $\chi(t)$ be a smooth function as in (3.2). If $h$ is a holomorphic section of a Hermitian vector bundle that does not vanish identically on any irreducible component of $X$ and $\chi_{\delta}=\chi(|h| / \delta)$, then

$$
\chi_{\delta} \omega \rightarrow \omega, \quad \bar{\partial} \chi_{\delta} \wedge \omega \rightarrow 0
$$

Proof Let $W$ be the zero set of $h$. Notice that $i_{*} \mathbf{1}_{X_{\text {sing }}} \omega=\mathbf{1}_{X_{\text {sing }}} i_{*} \omega=\mathbf{1}_{X_{\text {sing }}} \Omega \wedge R=$ $\Omega \wedge \mathbf{1}_{X_{\text {sing }}} R=0$ in view of Lemma 4.1. Thus $\mathbf{1}_{X_{\text {sing }}} \omega=0$, and hence $\mathbf{1}_{W} \mathbf{1}_{X_{\text {sing }}} \omega=0$. Since $\omega$ is smooth on $X_{\text {reg }}$ we have that $\mathbf{1}_{W} \mathbf{1}_{X_{\text {reg }}} \omega=0$. By simple computational rules, see, e.g., [10], we conclude that $\mathbf{1}_{W} \omega=0$. In view of (3.2) thus the first part of (4.11) follows. Notice that (4.4) implies that $(a-\bar{\partial}) R=0$, and by (4.8) thus $(a-\bar{\partial}) \omega=0$. Applying $(a-\bar{\partial})$ to the first limit in (4.11) now the second one follows.

\section{Koppelman Formulas on a Projective Variety}

Let $U^{\lambda}=\left|a_{1}\right|^{2 \lambda} U$ and $R^{\lambda}=1-\left|a_{1}\right|^{2 \lambda}+\bar{\partial}\left|a_{1}\right|^{2 \lambda} \wedge U$. Then $R^{\lambda}$ and $U^{\lambda}$ are as smooth as we may wish if $\operatorname{Re} \lambda$ is sufficiently large. In particular, $R^{\lambda}$ and $U^{\lambda}$ are well-defined currents. Moreover, they admit analytic continuations to $\operatorname{Re} \lambda>-\epsilon$, and the values at $\lambda=0$ are precisely $R$ and $U$, respectively, see [7]. 
Let $\rho$ be an integer. Following [3, Definition 1] we say that $H=\left(H_{k}^{\ell}\right)$ is a Hefer morphism for the complex $E_{\bullet} \otimes \mathcal{O}(\rho), a$, cf. (4.1), if $H_{k}^{\ell}$ are smooth sections of

$$
\mathcal{L}^{-k+\ell}\left(\operatorname{Hom}\left(E_{\zeta, k} \otimes \mathcal{O}_{\zeta}(\rho), E_{z, \ell} \otimes \mathcal{O}_{z}(\rho)\right)\right),
$$

$H_{k}^{\ell}=0$ for $k<\ell$, the term $\left(H_{\ell}^{\ell}\right)_{0}$ of bidegree $(0,0)$ is the identity $I_{E_{\ell}}$ on $\Delta$, and

$$
\nabla_{\eta} H_{k}^{\ell}=H_{k-1}^{\ell} a_{k}-a_{\ell+1}(z) H_{k}^{\ell+1},
$$

where $a_{k}$ stands for $a_{k}(\zeta)$. From [4, Lemma 2.5] we have

Lemma 5.1 Assume that $H$ is a Hefer morphism for the complex $E_{\bullet} \otimes \mathcal{O}(\rho)$, a. For $\operatorname{Re} \lambda \gg 0$, the form

$$
g^{\lambda}=a_{1}(z) H^{1} U^{\lambda}+H^{0} R^{\lambda}
$$

is a weight with respect to $\mathcal{O}(\rho)$.

Here $H^{1} U=\sum_{j} H_{j}^{1} U_{j}$ and $H^{0} R=\sum_{j} H_{j}^{0} R_{j}$.

Proposition 5.2 Let $F$ be holomorphic vector bundle over $\mathbb{P}^{N}$ and let $g$ be a weight with respect to $F$. Moreover, assume that $H$ is a Hefer morphism for $E_{\bullet} \otimes L^{\rho}$. For $\phi \in \mathcal{E}^{0, q}\left(X, F \otimes L^{\rho-N}\right)$ we have the Koppelman formula

$$
\begin{aligned}
\phi(z)= & \bar{\partial}_{z} \int_{\zeta}(H R \wedge g \wedge B)_{N} \wedge \phi+\int_{\zeta}(H R \wedge g \wedge B)_{N} \wedge \bar{\partial} \phi \\
& +\int_{\zeta}(H R \wedge g)_{N} \wedge \phi, \quad z \in X_{\text {reg }} .
\end{aligned}
$$

By blowing up $\mathbb{P}^{N} \times \mathbb{P}^{N}$ along the diagonal one can verify that $B$ is almost semimeromorphic. In view of Proposition 3.1 thus $(H R \wedge g \wedge B)_{N} \wedge \phi$ is a well-defined pseudomeromorphic current on $\mathbb{P}^{N} \times \mathbb{P}^{N}$. Formally (5.3) means that

$$
\phi=\bar{\partial} \pi_{*}\left((H R \wedge g \wedge B)_{N} \wedge \phi\right)+\pi_{*}\left((H R \wedge g \wedge B)_{N} \wedge \bar{\partial} \phi\right)+\pi_{*}\left((H R \wedge g)_{N} \wedge \phi\right),
$$

where $\pi$ is the projection $(\zeta, z) \mapsto z$.

Proof Since $g^{\lambda} \wedge g$ is a weight with respect to $\mathcal{O}(\rho) \otimes F$, and $a_{1}(z)=0$ when $z \in X$, from Proposition 2.2 we get (5.3) with $R^{\lambda}$ instead of $R$. In view of (the proof of) [6, Lemma 5.2], see also [9, Lemma 9.5], we can take $\lambda=0$ and so we get the proposition, keeping in mind that the product $B \wedge \tau$ can be defined as the value of $|\eta|^{2 \lambda} B \wedge \tau$ at $\lambda=0$ in view of $[6,(2.2)$ and (2.3)].

Let

$$
\mathcal{K} \phi=\pi_{*}\left((H R \wedge g \wedge B)_{N} \wedge \phi\right), \quad \mathcal{P} \phi=\pi_{*}\left((H R \wedge g)_{N} \wedge \phi\right) .
$$

Then we can write (5.3) as

$$
\phi=\bar{\partial} \mathcal{K} \phi+\mathcal{K} \bar{\partial} \phi+\mathcal{P} \phi
$$


for $z \in X_{\text {reg }}$.

In view of (4.10) we have that

$$
\mathcal{K} \phi(z)=\int_{X} k(\zeta, z) \wedge \phi(\zeta), \quad \mathcal{P} \phi(z)=\int_{X} p(\zeta, z) \wedge \phi(\zeta)
$$

where

$$
k= \pm \vartheta(g \wedge B \wedge H) \wedge \omega, \quad p= \pm \vartheta(g \wedge H) \wedge \omega .
$$

It is apparent from (5.6) that $\mathcal{K}$ and $\mathcal{P}$ are intrinsic integral operators on $X$. Locally they are precisely of the type in [6], so it follows that $\mathcal{K} \phi$ is smooth on $X_{\text {reg }}$ if $\phi$ is smooth. Moreover, from [6, Theorem 1.4] we get:

Theorem 5.3 Let $F$ be holomorphic vector bundle over $\mathbb{P}^{N}$ and let $g$ be a weight with respect to $F$ on $X$. Moreover, assume that $H$ is a Hefer morphism for $E_{\bullet} \otimes \mathcal{O}(\rho)$ and $\mathcal{K}$ and $\mathcal{P}$ are defined by (5.6). Then

$$
\begin{aligned}
& \mathcal{K}: \mathcal{A}^{k+1}\left(X, E \otimes L^{\rho-N}\right) \rightarrow \mathcal{A}^{k}\left(X, E \otimes L^{\rho-N}\right), \\
& \mathcal{P}: \mathcal{A}^{k}\left(X, E \otimes L^{\rho-N}\right) \rightarrow \mathcal{E}^{0, k}\left(\mathbb{P}^{N}, E \otimes \mathcal{O}(\rho-N)\right)
\end{aligned}
$$

and the global Koppelman formula (5.5) holds on $X$ for $\phi \in \mathcal{A}^{q}\left(X, F \otimes L^{\rho-N}\right)$.

\section{The Non-reduced Case}

Now assume that $i: X \rightarrow \mathbb{P}^{N}$ has pure dimension $n$ but is non-reduced. Then we still have an ideal sheaf $\mathcal{J}_{X} \subset \mathcal{O}_{\mathbb{P}^{N}}$ that has pure dimension $n$ but $\mathcal{J}_{X}$ is no longer radical, i.e., there are nilpotent elements. Still the structure sheaf of $X$ has the representation $\mathcal{O}_{X}=\mathcal{O}_{\mathbb{P}^{N}} / \mathcal{J}_{X}$. The underlying reduced space $X_{\text {red }}$ is associated with the radical ideal $\sqrt{\mathcal{J}_{X}}=\mathcal{J}_{X_{\text {red }}}$.

Let $X_{\text {reg }}$ be the subset of $X$ where $X_{\text {red }}$ is smooth and in addition $\mathcal{J}_{X}$ is CohenMacaulay. In a neighborhood $\mathcal{U}$ of a point $x$ in $X_{\text {reg }}$, which is an open dense subset of $X$, we can choose local coordinates $(z, w)$ such that $X_{\text {red }} \cap \mathcal{U}=\{w=0\}$. It turns out, see, e.g., [9], that there are monomials $1, w^{\alpha_{1}}, \ldots, w^{\nu-1}$ such that each $\phi$ in $\mathcal{O}_{X}$ has a unique representation

$$
\phi=\hat{\phi}_{0}(z) \otimes 1+\cdots+\hat{\phi}_{\nu-1}(z) \otimes w^{\nu-1} .
$$

Thus $\mathcal{O}_{X}$ has the structure of a free $\mathcal{O}_{X_{\text {red }}}$-module in $\mathcal{U}$.

We say that $\Phi$ in $\mathcal{E}_{\mathbb{P}^{N}}^{0, *}$ is in $\mathcal{K} e r i^{*}$ if in a neighborhood of each point in $X_{\text {reg }}$, $\Phi$ is in the subsheaf of $\mathcal{E}_{\mathbb{P}^{N}}^{0, *}$ generated by $\mathcal{J}_{X}, \overline{\mathcal{J}}_{X_{\text {red }}}$, and $d \overline{\mathcal{J}}_{X_{\text {red }}}$. As in the reduced case we define $\mathcal{E}_{X}^{0, *}=\mathcal{E}_{\mathbb{P}^{N}} / \mathcal{K}$ er $i^{*}$, and again it is independent of the choice of local embedding of $X$. It turns out that at each point in $X_{\text {reg }}$ and coordinates $(z, w)$ as above we have a unique representation (6.1) of $\phi$ in $\mathcal{E}_{X}^{0, *}$ where $\hat{\phi}_{j}$ are in $\mathcal{E}_{X_{\text {red }}}^{0, *}$. 
We define the sheaf of $(n, *)$-currents on $X$ as the dual of $\mathcal{E}_{X}^{0, n-*}$, so that such a current $\tau$ is represented by a $(N, N-n+*)$-current $i_{*} \tau$ in $\mathbb{P}^{N}$ that is annihilated by Ker $i^{*}$.

Basically all facts in Sect. 4 now hold verbatim, except for that one has to replace $X$ by $X_{\text {red }}$ in (4.5) and slightly modify the proof of Lemma 4.1. It follows from Lemma 4.1 that there is a current $\omega$ such that (4.8) holds. However, in the non-reduced case we give no meaning to that $\omega$ is almost semi-meromorphic and smooth on $X_{\text {reg }}$. The first part of (4.11) just means that $\mathbf{1}_{W} R=0$ and this follows from [9, Corollary 6.3]. The second part of (4.11) follows from the first part precisely as before.

Following [9] we can also make the construction of Koppelman formulas in Sect. 5 and define sheaves $\mathcal{A}^{*}$ on $X$ so that Theorem 5.3 holds.

Remark 4 Recall that a holomorphic differential operator $L$ is Noetherian with respect to the ideal $\mathcal{J}$ if $L \phi$ vanishes on $Z$ if $\phi \in \mathcal{J}$. As in the local case, cf. [6, Remark 6.6], there is a tuple $\mathcal{L}$ of global Noetherian operators on $\mathbb{P}^{N}$ with almost semi-meromorphic coefficients so that

$$
\omega \cdot \xi=\int_{Z} \mathcal{L} \xi
$$

cf. [8, Theorem 4.1 and Proposition 5.1].

\section{Global Solutions}

To begin with we consider a Hefer morphism, introduced in [3], for the complex $E_{\bullet} \otimes \mathcal{O}(\rho), a$ for large $\rho$. Let $E^{\prime}$ denote the complex of trivial bundles over $\mathbb{C}^{N+1}$ that we get from $E$, and let $A$ denote the corresponding mappings (which then formally are just the original matrices $a$ ). Let $\delta_{w-z}$ denote interior multiplication by

$$
2 \pi i \sum_{0}^{N}\left(w_{j}-z_{j}\right) \frac{\partial}{\partial w_{j}} .
$$

in $\mathbb{C}_{w}^{N+1} \times \mathbb{C}_{z}^{N+1}$.

Proposition 7.1 There exist $(k-\ell, 0)$-form-valued mappings

$$
h_{k}^{\ell}=\sum_{i j}\left(h_{k}^{\ell}\right)_{i j} e_{\ell i} \otimes e_{k j}^{*}: \mathbb{C}_{w}^{N+1} \times \mathbb{C}_{z}^{N+1} \rightarrow \operatorname{Hom}\left(E_{k}^{\prime}, E_{\ell}^{\prime}\right) \text {, }
$$

such that $h_{k}^{\ell}=0$ for $k<\ell, h_{\ell}^{\ell}=I_{E_{\ell}^{\prime}}$

$$
\delta_{z-w} h_{k}^{\ell}=h_{k-1}^{\ell} A_{k}(w)-A_{\ell+1}(z) h_{k}^{\ell+1},
$$

and the coefficients in the form $\left(h_{k}^{\ell}\right)_{i j}$ are homogeneous polynomials of degree $d_{k}^{j}-d_{\ell}^{i}-(k-\ell)$. 
Notice that

$$
\gamma_{j}=d \zeta_{j}-\frac{\bar{\zeta} \cdot d \zeta}{|\zeta|^{2}} \zeta_{j}
$$

is a projective form and that

$$
\nabla_{\eta} \gamma_{j}=2 \pi i\left(z_{j}-\alpha \zeta_{j}\right)
$$

Given $h_{k}^{\ell}$ in Proposition 7.1 we let $\tau^{*} h_{k}^{\ell}$ be the projective form we obtain by replacing $w$ by $\alpha \zeta$ and $d w_{j}$ by $\gamma_{j}$. We then have

$$
\nabla_{\eta} \tau^{*} h=\tau^{*}\left(\delta_{w-z} h\right)
$$

in light of (7.3) and (2.4). It is proved in [3] that if

$$
\kappa_{0}=\kappa_{0}(X)=\max d_{k}^{i}
$$

then

$$
H_{k}^{\ell}=\sum_{i j}\left(\tau^{*} h_{k}^{\ell}\right)_{i j} \wedge \alpha^{\kappa_{0}-d_{k}^{j}} e_{\ell, i} \otimes e_{k, j}^{*},
$$

is a Hefer morphism for (4.1) with $\rho=\kappa_{0}$. Clearly, $H$ is holomorphic in $z$.

Recall that $g=\alpha^{v}$ is a holomorphic weight with respect to $F=\mathcal{O}(v)$ for $v \geq 0$. From Proposition 5.2 we thus obtain explicit solutions to the $\bar{\partial}$-equation in $L^{\bar{\ell}}$ for $\ell \geq \kappa_{0}(X)-N$.

Proposition 7.2 Assume that $X$ is a possibly singular projective subvariety of $\mathbb{P}^{N}$ of pure dimension $n$, and $s \geq \kappa_{0}-N$. If $\phi$ is a $\bar{\partial}$-closed section in $\mathcal{A}^{q}\left(X, L^{s}\right), q \geq 1$, then

$$
\mathcal{K} \phi(z)=\int\left(H_{\kappa_{0}} R \wedge \alpha^{s-\kappa_{0}+N} \wedge B\right)_{N} \wedge \phi
$$

is a solution in $\mathcal{A}^{q-1}\left(X, L^{s}\right)$ to $\bar{\partial} u=\phi$.

Notice that $\kappa_{0}-N \leq \max \left(d_{k}^{i}-k\right) \leq \operatorname{reg} X-1$. Thus the proposition gives a weaker form of Theorem 1.1.

Remark 5 The associated operator $\mathcal{P}$ is precisely the operator in [4, Example 3.4] that realizes the surjectivity of (1.7). That is, if $\phi \in \mathcal{O}\left(X, L^{S}\right)$, then

$$
\phi(z)=\int\left(H R \wedge \alpha^{s-\kappa_{0}+N}\right)_{N} \wedge \phi
$$

is a global section of $\mathcal{O}(s) \rightarrow \mathbb{P}^{N}$ that coincides with $\phi$ on $X$. 
Example 2 Assume that $X$ is a complete intersection, i.e., $J$ is generated by homogeneous forms $a_{1}^{1}, \ldots, a_{1}^{p}$, of degrees $d^{1}, \ldots, d^{p}$, where $p=N-n$. Then the Koszul complex generated by $a_{1}^{j}$ provides a minimal free resolution, and it is then easy to see that $\kappa_{0}=d^{1}+\cdots+d^{p}$, cf. Sect. 8 below. Moreover, by the adjunction formula

$$
K_{X}=K_{\mathbb{P}^{N}} \otimes \mathcal{O}\left(d^{1}+\cdots+d^{p}\right)=L^{\kappa_{0}-N-1} .
$$

Here $K_{X}$ is the Grothendieck dualizing sheaf, which in this case is a line bundle that is generated by $\omega=\omega_{0}$. When $X$ is smooth $K_{X}$ is just the usual canonical bundle. If we define $(n, q)$-forms as $(0, q)$-forms with values in $K_{X}$, then Proposition 7.2 gives an explicit realization of the vanishing

$$
H^{n, q}\left(X, L^{\ell}\right)=0, \quad 1 \leq q \leq n, \quad \ell \geq 1
$$

If $X$ is smooth this follows precisely from Kodaira's theorem, since $L$ is strictly positive on $X$.

For the proof of Theorem 1.1 we must make a more careful analysis of the kernels. Let us introduce the notation

$$
\kappa_{q}=\kappa_{q}(X)=\max _{\ell \leq N-q} d_{\ell}^{i}
$$

Notice that

$$
\kappa_{q}-N \leq=\max _{\ell \leq N-q}\left(d_{\ell}^{i}-(N-\ell)-\ell\right) \leq \operatorname{reg} X-1-q .
$$

Proof of Theorem 1.1 Notice that the section $h(\zeta, z)=\zeta \cdot \bar{z} /|z|^{2}$ of $\mathcal{O}_{\zeta}(1) \otimes \mathcal{O}_{z}(-1)$ is non-vanishing on $\Delta$. Let $\chi(t)$ be a cutoff function as before and let

$$
\chi_{\delta}:=\chi\left(|h|^{2} / \delta\right)=\chi\left(|\bar{\zeta} \cdot z|^{2} /|z|^{2}|\zeta|^{2} \delta\right) .
$$

Here $|h|$ denote the natural norm of the section $h$, whereas in the last term || denotes norm of points in $\mathbb{C}^{N+1}$. For small $\delta, \chi_{\delta}$ is identically 1 in a neighborhood of $\Delta$ and thus

$$
g^{\delta}:=\chi_{\delta}-\bar{\partial} \chi_{\delta} \wedge B
$$

is a smooth weight (with respect to the trivial line bundle). For fixed $z, g^{\delta}$ vanishes in a neighborhood of the hyperplane $h=0$, and therefore

$$
\alpha^{-r} \wedge g^{\delta}
$$

is a smooth weight with respect to $\mathcal{O}(-r)$ for any $r$, though not holomorphic in $z$. Now fix $q \geq 1$ and let $t=s-q$. In particular, for $t \geq \kappa_{q}-N$ and $\phi \in \mathcal{A}^{q}\left(X, L^{t}\right)$, $q \geq 1$, with $\bar{\partial} \phi=0$ we have the formula

$$
\phi(z)=\bar{\partial} \int_{\zeta}\left(H R \wedge \alpha^{t-\kappa_{0}+N} \wedge g^{\delta} \wedge B\right)_{N} \wedge \phi
$$




$$
+\int_{\zeta}\left(H R \wedge \alpha^{t-\kappa_{0}+N} \wedge g^{\delta} \wedge \phi\right)_{N} \wedge \phi=\bar{\partial} \mathcal{K}^{\delta} \phi+\mathcal{P}^{\delta} \phi .
$$

We claim that $\mathcal{K}^{\delta} \phi$ tends to a current $\mathcal{K} \phi$ in $\mathcal{A}^{q-1}\left(X, L^{t}\right)$ and that $\mathcal{P}^{\delta} \phi \rightarrow 0$. Taking this for granted, the theorem follows in view of (7.7).

To settle the claim we first consider the expression for $\mathcal{P}^{\delta} \phi$ in (7.9). Since $\phi$ has bidegree $(0, q)$ only components $H_{N-\ell} R_{N-\ell}$ with $\ell \geq q$ can occur in the integral. Thus the total power of $\alpha$ is

$$
\kappa_{0}-d_{N-\ell}^{i}+t-\kappa_{0}+N \geq \kappa_{0}-d_{N-\ell}^{i}+\kappa_{q}-N-\kappa_{0}+N=\kappa_{q}-d_{N-\ell}^{i} \geq 0
$$

in view of (7.6). Thus

$$
\mathcal{P}^{\delta} \phi(z)=\sum_{\ell \geq q} \int_{X} \omega_{n-\ell} \wedge \phi_{0, q} \wedge \vartheta\left(\xi_{\ell} \wedge\left(\chi_{\delta}-\bar{\partial} \chi_{\delta} \wedge B\right)\right)
$$

where $\xi_{\ell}$ are smooth and holomorphic in $z$. Since $q \geq 1$ we need some antiholomorphic differentials with respect to $z$ and they must come from $\bar{\partial} \chi_{\delta} \wedge B$; hence we can forget about $\chi_{\delta}$. Since $\chi_{\delta}=1$ in a neighborhood of the diagonal, we can consider $B$ as smooth. Thus we have to verify that

$$
\omega \wedge \phi \wedge \bar{\partial} \chi_{\delta} \rightarrow 0, \quad \delta \rightarrow 0
$$

Since $X$ is irreducible, $h=0$ has positive codimension on $X$, and if $\phi$ is smooth thus (7.10) holds in view of Lemma 4.3. If $\phi$ is in $\mathcal{A}^{q}$, then it is in $\operatorname{Dom}_{X}$, cf. [6,9], and then (7.10) follows from (the proofs of) [6, Lemma 4.1] and [9, Lemma 8.4]. In fact, (7.10) can be reformulated as $\mathbf{1}_{h=0} \bar{\partial}(\omega \wedge \phi)=0$. We conclude that $\mathcal{P}^{\delta} \phi \rightarrow 0$. Notice now that $B \wedge B=0$ so that

$$
\mathcal{K}^{\delta} \phi=\int_{\zeta} \chi_{\delta}\left(H R \wedge \alpha^{t-\kappa_{0}+N} \wedge B\right)_{N} \wedge \phi
$$

It is proved in [6,9] that $\left(H R \wedge \alpha^{t-\kappa_{0}+N} \wedge B\right)_{N} \wedge \phi$ is in the space $\mathcal{W}^{X \times \mathbb{P}^{N}}$, and this implies that

$$
\chi_{\delta}\left(H R \wedge \alpha^{t-\kappa_{0}+N} \wedge B\right)_{N} \wedge \phi \rightarrow\left(H R \wedge \alpha^{t-\kappa_{0}+N} \wedge B\right)_{N} \wedge \phi
$$

It follows that

$$
\mathcal{K}^{\delta} \phi \rightarrow \mathcal{K} \phi=\int_{\zeta}\left(H R \wedge \alpha^{t-\kappa_{0}+N} \wedge B\right)_{N} \wedge \phi
$$




\subsection{Examples with Negative Curvature}

We now turn our attention to the case of negative curvature. We define a Hefer morphism from $h_{k}^{\ell}$ in Proposition 7.1 by replacing $w$ by $\zeta, z$ by $\beta z$, and $d w_{j}$ by the $\gamma_{j}$ from Proposition 2.4. The morphism so obtained is a Hefer morphism for (4.1) (i.e., for $E_{\bullet} \otimes \mathcal{O}(\rho), a$ with $\left.\rho=0\right)$. This is verified in the same way as [3, Proposition 4.4].

This time $H$ is not holomorphic in $z$ but in $\zeta$ instead. Let $\delta$ be the depth of the ring $S / J$. This is a number, $0 \leq \delta \leq n$, and choosing (1.4) minimal, (4.1) will end up at $k=N-\delta$, which means that $R=R_{N-n}+\cdots+R_{N-\delta}$. The variety $X$ is Cohen-Macaulay precisely when $\delta=n$.

From the Koppelman formula we get solutions to $\bar{\partial}$ (representation of the cohomology in the smooth case) for $(0, q)$-forms $\phi$ with values in $\mathcal{O}(\ell)$ for $\ell \leq-N$ and thus solutions as soon as the obstruction term

$$
\int\left(H R \wedge \beta^{-N-\ell}\right)_{N} \wedge \phi
$$

vanishes. Notice that $H R$ has degree at $\operatorname{most} N-\delta$ in $d \bar{\zeta}$ since $\beta$ and $\gamma_{j}$ only contain holomorphic differentials with respect to $\zeta$. Therefore (7.11) must vanish if $N-\delta+q<$ $N$, i.e., $0 \leq q \leq \delta-1$.

Theorem 7.3 Assume that $X$ is a subvariety of $\mathbb{P}^{N}$ of pure dimension $n$ and $\ell \leq-N$. Then for any $\bar{\partial}$-closed $(0, q)$-form $\phi \in \mathcal{A}_{q}\left(X, L^{\ell}\right), 0<q \leq \delta-1$,

$$
\psi(z)=\int\left(H R \wedge \beta^{-N-\ell} \wedge B\right)_{N} \wedge \phi
$$

is a solution in $\mathcal{A}^{q-1}\left(X, L^{\ell}\right)$ to $\bar{\partial} \psi=\phi$.

We thus have an explicit proof of the vanishing $H^{0, q}(X, \mathcal{O}(\ell))=0$ for $0 \leq q \leq$ $\delta-1, \ell \leq-N$.

\section{Global Complete Intersections}

Let us compute the resulting formulas in case $i: X \rightarrow \mathbb{P}^{N}$ is a global complete intersection as in Example 2. Let $f_{1}, \ldots, f_{p}, f_{j}=a_{1}^{j}$, be our given homogeneous forms of degrees $d^{j}$ from Example 2, and recall that $p=N-n$. Assume that $E_{0}$ is the trivial line bundle and let

$$
E=E^{1} \otimes \mathcal{O}\left(-d^{1}\right) \oplus \cdots \oplus E^{p} \otimes \mathcal{O}\left(-d^{p}\right)
$$

where $E^{j}$ are trivial line bundles. Let $e_{j}$ be basis elements for $E^{j}$ and let $e_{j}^{*}$ be the dual basis elements. We take

$$
E_{k}=\Lambda^{k} E=\sum_{|I|=k}^{\prime} \mathcal{O}\left(-\left(d^{I_{1}}+\cdots+d^{I_{k}}\right)\right) E^{I_{1}} \otimes \cdots \otimes E^{I_{k}}
$$


and $a_{k}: E_{k} \rightarrow E_{k-1}$ as interior multiplication by $f=\sum f_{j} e_{j}^{*}$. Now

$$
\sigma=\sum_{j} \frac{\overline{f_{j}(z)}}{|z|^{2 d^{j}}} e_{j} /\|f\|^{2}
$$

is the section of $E$ with minimal norm such that $f \cdot \sigma=1$ outside $Z$, if $\|f\|=|f|_{E^{*}}$. Moreover,

$$
U=\left.\|f\|^{2 \lambda} \sum_{k=1}^{m} \sigma \wedge(\bar{\partial} \sigma)^{k-1}\right|_{\lambda=0}
$$

and

$$
R=1-\|f\|^{2 \lambda}+\left.\bar{\partial}\|f\|^{2 \lambda} \wedge \sum_{k=1}^{m} \sigma \wedge(\bar{\partial} \sigma)^{k-1}\right|_{\lambda=0},
$$

cf. Sect. 5 and [2]; here $\left.\right|_{\lambda=0}$ means evaluation at $\lambda=0$ after analytic continuation.

Since $\operatorname{codim} Z=p$ the resulting residue current $R$ just consists of the term $R_{p}$; it coincides with the classical Coleff-Herrera product

$$
\bar{\partial} \frac{1}{f_{p}} \wedge \cdots \wedge \bar{\partial} \frac{1}{f_{1}} \wedge e_{1} \wedge \cdots \wedge e_{p} .
$$

We now compute Hefer morphisms for the Koszul complex. Let $\tilde{h}_{j}(w, z)$ be $(1,0)$ forms in $\mathbb{C}^{n+1} \times \mathbb{C}^{n+1}$ of polynomial degrees $d^{j}-1$ such that

$$
\delta_{w-z} \tilde{h}_{j}=f_{j}(w)-f_{j}(z)
$$

and let $h_{j}=\tau^{*} \tilde{h}_{j}$. We only have to care about $k \leq p$ so $\kappa_{0}=d^{1}+\cdots+d^{p}$. Then

$$
H_{k}^{\ell}=\sum_{|I|=\ell|J|=k-\ell}^{\prime} \sum_{J_{1}}^{\prime} \pm \cdots \wedge h_{J_{k-\ell}} \wedge e_{I} \otimes e_{I J}^{*} \wedge \alpha^{\kappa_{0}-\left(d^{J_{1}}+\cdots+d^{J_{k-\ell}}+d^{I_{1}}+\cdots+d^{I_{k}}\right)}
$$

is a Hefer morphism. The components of most interest for us are $H_{k}^{0}$ and $H_{k}^{1}$. Since

$$
H_{k}^{0}=\sum_{|J|=k}^{\prime} \pm h_{J_{1}} \wedge \cdots \wedge h_{J_{k}} \wedge e_{J}^{*} \wedge \alpha^{\kappa_{0}-\left(d^{J_{1}}+\cdots+d^{J_{k}}\right)}
$$

it can be more compactly written formally as

$$
H_{k}^{0}=\alpha^{\kappa_{0}} \wedge\left(\delta_{h}\right)_{k},
$$

where $\delta_{h}$ denotes formal interior multiplication with

$$
h=\sum \alpha^{-d^{j}} \wedge h_{j} \wedge e_{j}^{*}
$$


and $\left(\delta_{h}\right)_{k}=\left(\delta_{h}\right)^{k} / k !$. In the same way

$$
H_{k}^{1}=\alpha^{\kappa_{0}} \wedge N\left(\delta_{h}\right)_{k-1},
$$

where

$$
N=\sum_{j} \alpha^{-d^{j}} e_{j} \otimes e_{j}^{*}
$$

Our description of $U, H_{k}^{1}$, etc., is just to illustrate what these currents look like in the complete intersection case since they play a rule in the proofs above. As we have seen, however, in the final Koppelman formula only the term

$$
H_{p}^{0} R_{p}=h_{1} \wedge \cdots \wedge h_{p} \wedge \bar{\partial} \frac{1}{f_{p}} \wedge \cdots \wedge \bar{\partial} \frac{1}{f_{1}}
$$

of $H R$ occurs. It follows that the operator $\mathcal{K}$ in Proposition 7.2, with

$$
\kappa=s+N-\left(d^{1}+\cdots+d^{p}\right)
$$

has the more explicit form

$$
\mathcal{K} \phi(z)=\int\left(\alpha^{\kappa} \wedge B \wedge h_{1} \wedge \cdots \wedge h_{p}\right)_{N} \wedge \bar{\partial} \frac{1}{f_{p}} \wedge \cdots \wedge \bar{\partial} \frac{1}{f_{1}} \wedge \phi
$$

and the operator $\mathcal{P}$ in Remark 5 is

$$
\mathcal{P} \phi(z)=\int\left(\alpha^{\kappa} \wedge h_{1} \wedge \cdots \wedge h_{p}\right)_{N} \wedge \bar{\partial} \frac{1}{f_{p}} \wedge \cdots \wedge \bar{\partial} \frac{1}{f_{1}} \wedge \phi .
$$

Proposition 8.1 Assume that the projective space $i: X \rightarrow \mathbb{P}^{N}$ of codimension $p$ is defined by the homogeneous forms $f_{j}$ on $\mathbb{C}^{N+1}$ of degree $d^{j}, j=1, \ldots, p$, and assume that $s \geq d^{1}+\cdots+d^{p}-N$. For $\phi \in \mathcal{E}^{0, k}\left(X, L^{s}\right)$, or $\phi \in \mathcal{A}^{k}\left(X, L^{s}\right)$, we have the Koppelman formula (5.5) with $\mathcal{K}$ and $\mathcal{P}$ defined by (8.3) and (8.4), respectively. Moreover, $\mathbb{P}$ vanishes if $k \geq 1$.

Remark 6 In [23] similar Koppelman formulas are obtained on a, not necessarily reduced, global complete intersection $X$ for $(0, *)$-forms with values in $L^{d^{1}+\cdots+d^{p}-N-1}$ in the notation from Example 2. The authors construct Koppelman formulas on homogeneous subvarieties of $\mathbb{C}^{N+1}$, keep track of homogeneities and so obtain Koppelman formulas on $X$. They use the same definition of $\bar{\partial}$ as we do. However, they only consider solutions to $\bar{\partial} u=\phi$ on $X_{\text {reg }}$ when $\phi$ is smooth on $X$ and satisfies a condition $(*)$ that in general is stronger than $\bar{\partial} \phi=0$. There is no discussion whether their solution has some meaning as a current across $X_{\text {sing }}$. The condition (*) on $\phi$ means that (locally) there is a smooth extension $\Phi$ to ambient space such that $\bar{\partial} \Phi$ is in $\mathcal{E} \mathcal{J}$. Clearly this implies that $\bar{\partial} \phi=0$ on $X$ but in general the converse 
does not hold. In fact, consider a reduced hypersurface $X=\{a=0\} \subset \mathbb{C}^{n+1}$ so that $\mathcal{J}=(a)$. Then $(*)$ means that there is an extension $\Phi$ such that $\bar{\partial} \Phi=\xi a$ for some smooth form $\xi$. Then $0=a \bar{\partial} \xi$ and thus $\bar{\partial} \xi=0$; hence $\xi=\bar{\partial} \eta$ for some smooth $\eta$. Now $\Phi-a \eta$ is $\bar{\partial}$-closed and therefore there is a smooth solution to $\bar{\partial} \Psi=\Phi-a \eta$. It follows that $\psi=i^{*} \Psi$ is a smooth solution to $\bar{\partial} \psi=\phi$. However, it is well known that there are smooth $\phi$ with $\bar{\partial} \phi=0$ such that $\bar{\partial} \psi=\phi$ has no smooth solution, see, e.g., [6, Example 1.1].

\subsection{The Reduced Case}

Let us consider a more intrinsic-looking representation of $\mathcal{K}$ and $\mathcal{P}$ as in (5.6) and (5.7). In order to avoid a Noetherian operator, cf. Remark 4, let us in addition assume that $X$ is reduced. Let $A_{1}, \ldots, A_{p}$ be holomorphic vector fields on $\mathbb{C}^{N+1}$ such that

$$
\delta_{A_{p}} \cdots \delta_{A_{1}} d f_{1} \wedge \cdots \wedge d f_{p}=(2 \pi i)^{p}
$$

or equivalently,

$$
d f_{1} \wedge \cdots d f_{p} \wedge \delta_{A_{p}} \cdots \delta_{A_{1}}\left(d \zeta_{0} \wedge \cdots \wedge d \zeta_{N}\right)=(2 \pi i)^{p} d \zeta_{0} \wedge \cdots \wedge d \zeta_{N}
$$

Notice that since $\delta_{\zeta}$ anti-commutes with $\delta_{A_{j}}$,

$$
\omega^{\prime}:=\delta_{A_{p}} \cdots \delta_{A_{1}} \Omega
$$

is a projective form. Following the proof of [4, Proposition 6.3] we see that $\omega^{\prime}$ is a representative for the structure form on $X$, that is,

$$
\omega=\omega_{0}=i^{*} \omega^{\prime}
$$

Example 3 Let

$$
\delta_{A}=\delta_{A_{1}} \cdots \delta_{A_{p}}, \quad \bar{\partial} \frac{1}{f}=\bar{\partial} \frac{1}{f_{p}} \wedge \cdots \bar{\partial} \frac{1}{f_{1}} .
$$

Then

$$
\delta_{A} \xi_{N}=\delta_{A}(\vartheta(\xi) \wedge \Omega)= \pm \vartheta(\xi) \wedge \omega^{\prime}
$$

In view of (5.7) we thus have that

$$
\mathcal{K} \phi= \pm \int_{X} \delta_{A}\left(\alpha^{\kappa} \wedge B \wedge h\right)_{N} \wedge \phi, \quad \mathcal{P} \phi= \pm \int_{X} \delta_{A}\left(\alpha^{\kappa} \wedge h\right)_{N} \wedge \phi
$$

for $\phi \in \mathcal{A}^{q}\left(X, L^{s}\right), s=\kappa+d^{1}+\cdots+d^{p}-N, \kappa \geq 0$. 


\subsection{Explicit Formulas for a Curve in $\mathbb{P}^{N}$}

Following [19] we will now describe how one can find an especially simple expression for the kernel $k$ when $X$ is a curve. Applying $\delta_{\eta}$ to

$$
\left(\alpha^{\kappa} \wedge B \wedge h\right)_{N}=\vartheta\left(\alpha^{\kappa} \wedge B \wedge h\right) \wedge \Omega
$$

cf. (4.9), we get

$$
\delta_{\eta}\left(\alpha^{\kappa} \wedge B \wedge h\right)_{N}= \pm \vartheta\left(\alpha^{\kappa} \wedge B \wedge h\right) \wedge \delta_{\eta} \Omega
$$

We claim that

$$
\delta_{\eta}\left(\alpha^{\kappa} \wedge B \wedge h\right)_{N}=\left(\alpha^{\kappa} \wedge h\right)_{N-1}
$$

on $X \times X$. In fact, consider the weight $g=\alpha^{\kappa} \wedge g^{\lambda}$, cf. (5.2). From (2.2), keeping $z$ on $X$ and taking $\lambda=0$ we get

$$
\nabla_{\eta}\left(\alpha^{\kappa} \wedge B \wedge h\right) \wedge R=\alpha^{\kappa} \wedge h \wedge R
$$

outside the diagonal. Identifying terms of degree $N-1$ in $d \zeta$ we get

$$
\delta_{\eta}\left(\alpha^{\kappa} \wedge B \wedge h\right)_{N} \wedge R=\left(\alpha^{\kappa} \wedge h\right)_{N-1} \wedge R
$$

By the generalized Poincaré-Lelong formula $R \wedge d f=(2 \pi i)^{p}[X]$ we can conclude that (8.10) holds on $X \times X$. Combining (8.9) and (8.10) we get that

$$
\pm \vartheta\left(\alpha^{\kappa} \wedge B \wedge h\right) \wedge \delta_{\eta} \Omega=\left(\alpha^{\kappa} \wedge h\right)_{N-1}
$$

on $X \times X$.

Let us now compute the kernel $k$ where $X$ is parametrized by $\left[\zeta_{0}, \zeta_{1}\right]$. Let $\delta_{\zeta_{j}}$ denote interior multiplication by $\partial / \partial \zeta_{j}$. Notice that

$$
\delta_{\eta} \delta_{\zeta_{2}} \cdots \delta_{\zeta_{N}} \Omega=2 \pi i\left(\zeta_{1} z_{0}-\zeta_{0} z_{1}\right)
$$

If we apply $\delta_{\zeta_{2}} \cdots \delta_{\zeta_{N}}$ to $(8.11)$ we get

$$
\pm \vartheta\left(\alpha^{\kappa} \wedge B \wedge h\right)=\frac{1}{2 \pi i} \frac{\delta_{\zeta_{2}} \cdots \delta_{\zeta_{N}}\left(\alpha^{\kappa} \wedge h\right)_{N-1}}{\zeta_{1} z_{0}-\zeta_{0} z_{1}} .
$$

Notice that the denominator on the right-hand side is smooth. Recalling that $k(\zeta, z)=$ $\pm \vartheta\left(\alpha^{\kappa} \wedge B \wedge h\right) \wedge \omega$ on $X \times X$, cf. (5.7), we get from (8.12) and (8.6), cf. (8.7),

Proposition 8.2 With the notation above we have the explicit formula

$$
k(\zeta, z)=\frac{1}{2 \pi i} \frac{\delta_{\zeta_{2}} \cdots \delta_{\zeta_{N}}\left(\alpha^{\kappa} \wedge h\right)_{N-1}}{\zeta_{1} z_{0}-\zeta_{0} z_{1}} \delta_{A} \Omega
$$

where $X$ is parametrized by $\left[\zeta_{0}, \zeta_{1}\right]$. 


\subsection{Curves in $\mathbb{P}^{2}$}

Assume now that $N=2$ and $X=\{f=0\}$, where $f$ is a $d$-homogeneous form. Thus $s=\kappa+d-2$. Let $\tilde{h}(w, z)=h_{0} d w_{0}+h_{1} d w_{1}+h_{2} d w_{2}$ be a Hefer form for $f$; i.e., $h_{\ell}$ are $d-1$-homogeneous and

$$
2 \pi i \sum_{\ell=0}^{2} h_{\ell}(w, z)\left(w_{\ell}-z_{\ell}\right)=f(w)-f(z) .
$$

Notice that for degree reasons,

$$
\left(\alpha^{\kappa} \wedge h\right)_{1}=\alpha_{0}^{\kappa}\left(h_{0}\left(\alpha_{0} \zeta, z\right) \gamma_{0}+h_{1}\left(\alpha_{0} \zeta, z\right) \gamma_{1}+h_{2}\left(\alpha_{0} \zeta, z\right) \gamma_{2}\right)
$$

In what follows, for simplicity, let us right $\alpha$ rather than $\alpha_{0}$. If $\partial f / \partial \zeta_{2}$ is generically non-vanishing on $X$ and

$$
A=\frac{2 \pi i}{\partial f / \partial \zeta_{2}} \frac{\partial}{\partial \zeta_{2}}
$$

then $\delta_{A} d f=2 \pi i$ (generically) on $X$. We get

$$
k(\zeta, z)=\frac{1}{2 \pi i} \frac{\zeta_{0} d \zeta_{1}-\zeta_{1} d \zeta_{0}}{\zeta_{1} z_{0}-\zeta_{0} z_{1}} \frac{2 \pi i \delta_{\zeta_{2}}\left(\alpha^{\kappa} \wedge h\right)_{1}}{\partial f / \partial \zeta_{2}}
$$

Notice furthermore that

$$
\delta_{\zeta_{2}}\left(\alpha^{\kappa} \wedge h\right)_{1}=\alpha^{\kappa}\left(h_{2}(\alpha \zeta, z)-\frac{\bar{\zeta}_{2}}{|\zeta|^{2}} \sum_{j=0}^{2} h_{j}(\alpha \zeta, z) \zeta_{j}\right)
$$

Proposition 8.3 With the notation above we have

$$
k(\zeta, z)=\frac{1}{2 \pi i} \frac{\zeta_{0} d \zeta_{1}-\zeta_{1} d \zeta_{0}}{\zeta_{1} z_{0}-\zeta_{0} z_{1}} \frac{2 \pi i \alpha^{\kappa}}{\partial f / \partial \zeta_{2}}\left(h_{2}(\alpha \zeta, z)-\frac{\bar{\zeta}_{2}}{|\zeta|^{2}} \sum_{j=0}^{2} h_{j}(\alpha \zeta, z) \zeta_{j}\right)
$$

The second term in the brackets actually cancels out the singularity if $X$ is smooth.

Proposition 8.4 If $X$ is smooth and $\left[\zeta_{0}, \zeta_{1}\right]$ are local homogeneous coordinates, then

$$
k(\zeta, z)=\frac{1}{2 \pi i} \frac{\zeta_{0} d \zeta_{1}-\zeta_{1} d \zeta_{0}}{\zeta_{1} z_{0}-\zeta_{0} z_{1}} \frac{\alpha^{\kappa} 2 \pi i h_{2}(\alpha \zeta, z)}{\partial f / \partial \zeta_{2}}+\cdots,
$$

where $\cdots$ is smooth and holomorphic in $z$.

Notice that $2 \pi i h_{2}(\alpha \zeta, z)=\partial f / \partial \zeta_{2}$ on the diagonal. 
Proof Differentiating (8.14) with respect to $w_{j}$ gives

$$
2 \pi i h_{j}(w, z)=\frac{\partial f}{\partial w_{j}}(w)-2 \pi i \sum_{\ell} \frac{\partial h_{\ell}}{\partial w_{j}}\left(w_{\ell}-z_{\ell}\right) .
$$

Since $f$ is $d$-homogeneous,

$$
\sum_{j=0}^{2} \zeta_{j} \frac{\partial f}{\partial \zeta_{j}}=d \cdot f(\zeta)
$$

We conclude that

$$
\sum_{0}^{2} \zeta_{j} \tilde{h}_{j}\left(\alpha \zeta_{j}, z\right)=d \alpha^{d-1} \cdot f(\zeta)+\sum_{0}^{2} b_{\ell}(\zeta, \alpha \zeta, z)\left(\alpha \zeta_{\ell}-z_{\ell}\right)
$$

where $b_{\ell}(\zeta, w, z)$ are holomorphic, $d-2$-homogeneous in $(w, z)$ and 1-homogeneous in $\zeta$. Since $\zeta$ is on $X, f(\zeta)=0$. We thus get (8.18) where

$$
\cdots=\frac{1}{2 \pi i} \frac{\zeta_{0} d \zeta_{1}-\zeta_{1} d \zeta_{0}}{\zeta_{1} z_{0}-\zeta_{0} z_{1}} B
$$

and

$$
B=\frac{\bar{\zeta}_{2}}{|\zeta|^{2}} \alpha^{\kappa} \sum_{0}^{2} b_{j}(\zeta, \alpha \zeta, z)\left(\alpha \zeta_{j}-z_{j}\right)
$$

Without loss of generality we may assume that $\zeta_{0}=z_{0}=1$ and that $\zeta_{1}$ is a local coordinate on $X$ so that $\zeta^{\prime}=g\left(\zeta_{1}\right)$. Since $\alpha=1$ on the diagonal we then have that $B=B^{\prime}\left(\zeta_{1}-z_{1}\right)$ where $B^{\prime}$ is holomorphic in $z$. After homogenization we get that $B=B^{\prime \prime}\left(z_{0} \zeta_{1}-z_{1} \zeta_{0}\right)$, where $B^{\prime \prime}$ is holomorphic in $z$. Thus the proposition follows.

Corollary 8.5 If $\zeta_{1}$ is a local coordinate, then taking $\zeta_{1}=\tau, z_{1}=t$, and $\zeta_{0}=z_{0}=1$, we get

$$
k(\tau, t)=\frac{1}{2 \pi i} \frac{d \tau}{\tau-t} \frac{\left.\alpha^{\kappa} 2 \pi i h_{2}\left(1, \tau, \zeta_{2} ; 1, t, z_{2}\right)\right)}{\partial f / \partial \zeta_{2}}+\cdots,
$$

where $\cdots$ is smooth and holomorphic in $t$.

Example 4 If $f(z)=z_{0}^{3}+z_{1}^{3}+z_{2}^{3}$, then $X$ is a smooth surface of genus 1 . We can take

$$
\tilde{h}(z, w)=\sum_{j=0}^{2}\left(z_{j}^{2}+z_{j} w_{j}+w_{j}^{2}\right) d w_{j} / 2 \pi i .
$$


Then, where $\left[z_{0}, z_{1}\right]$ are homogeneous coordinates,

$$
\begin{aligned}
k(\zeta, z)= & \alpha^{\kappa} \frac{1}{2 \pi i} \frac{\zeta_{0} d \zeta_{1}-\zeta_{1} d \zeta_{0}}{\zeta_{1} z_{0}-\zeta_{0} z_{1}} \frac{1}{3 \zeta_{0}^{2}}\left[z_{2}^{2}+\alpha z_{2} \zeta_{2}+\alpha^{2} \zeta_{2}^{2}\right. \\
& \left.-\frac{\bar{\zeta}_{2}}{|\zeta|^{2}}\left(z_{0}^{2} \zeta_{0}+z_{1}^{2} \zeta_{1}+z_{2}^{2} \zeta_{2}+\alpha\left(z_{0} \zeta_{0}^{2}+z_{1} \zeta_{1}^{2}+z_{2} \zeta_{2}^{2}\right)\right)\right]
\end{aligned}
$$

Even if $X$ is not smooth, by the same argument, one can identify the principal term of the kernel $k$.

Example 5 The curve $X=\left\{z_{1}^{3}-z_{2}^{2} z_{0}=0\right\}$ has a cusp singularity at [1,0,0] and is smooth elsewhere. It is globally parametrized by

$$
\mathbb{P}^{1} \rightarrow X \subset \mathbb{P}^{2}, \quad\left[t_{0}, t_{1}\right] \mapsto\left[t_{0}^{3}, t_{1}^{2} t_{0}, t_{1}^{3}\right]
$$

We can choose the Hefer form

$$
2 \pi i \tilde{h}(w, z)=z_{2}^{2} d w_{0}-\left(z_{1}^{2}+z_{1} w_{1}+w_{1}^{2}\right) d w_{1}+\left(z_{2}+w_{2}\right) w_{0} d w_{2}
$$

By formula (8.17) we can now express $k$ completely in terms of the parameters $\left[t_{0}, t_{1}\right]$ and $\left[\tau_{0}, \tau_{1}\right]$. However, we restrict to considering the principal term. Since we are primarily interested in the singularity, we consider the standard affinization where $z_{0}=\zeta_{0}=1$. By the recipe above, then

$$
k=\frac{1}{2 \pi i} \frac{d \zeta_{1}}{\zeta_{1}-z_{1}} \alpha^{\kappa} \frac{z_{2}+\alpha \zeta_{2}}{2 \zeta_{2}}+\cdots
$$

In this case $\cdots$ is not smooth but at least the singularity is smaller than in the leading term. Since $\alpha-1=\mathcal{O}\left(\zeta_{1}-z_{1}\right)+\mathcal{O}\left(\zeta_{2}-z_{2}\right)$, we can delete $\alpha$ as well in the leading term in (8.20). We then have

$$
k=\frac{1}{2 \pi i} \frac{\left(\zeta_{2}^{2}-z_{2}^{2}\right) d \zeta_{1}}{\left(\zeta_{1}-z_{1}\right)\left(\zeta_{2}-z_{2}\right) 2 \zeta_{2}}+\cdots
$$

Letting $t=t_{1} / t_{0}$ and $\tau=\tau_{1} / \tau_{0}$ we are then left with

$$
k=\frac{1}{2 \pi i} \frac{\left(\tau^{6}-t^{6}\right) d \tau}{\left(\tau^{2}-t^{2}\right)\left(\tau^{3}-t^{3}\right) \tau^{2}}+\cdots
$$

where the leading term is precisely the kernel in the last example in [6, Sect. 8].

Acknowledgements The basic idea of this paper was used by P. Helgesson already in 2010; he skillfully worked out the details in the special but nontrivial case when $X$ is a smooth Riemann surface in $\mathbb{P}^{2}$, [19]. We are grateful to Helgesson for valuable discussions on these matters. We also would like to thank the referee for careful reading and pointing out several mistakes. Funding was provided by Vetenskapsrådet. 
Open Access This article is distributed under the terms of the Creative Commons Attribution 4.0 International License (http://creativecommons.org/licenses/by/4.0/), which permits unrestricted use, distribution, and reproduction in any medium, provided you give appropriate credit to the original author(s) and the source, provide a link to the Creative Commons license, and indicate if changes were made.

\section{References}

1. Andersson, M.: Integral representation with weights I. Math. Ann. 326, 1-18 (2003)

2. Andersson, M.: Residue currents and ideals of holomorphic functions. Bull. Sci. Math. 128, 481-512 (2004)

3. Andersson, M., Götmark, E.: Explicit representation of membership of polynomial ideals. Math. Ann. 349, 345-365 (2011)

4. Andersson, M., Nilsson, L.: Division formulas on projective varieties. Math. Z. 284, 575-593 (2016)

5. Andersson, M., Samuelsson, H.: Koppelman formulas and the $\bar{\partial}$-equation on an analytic space. J. Funct. Anal. 261, 777-802 (2011)

6. Andersson, M., Samuelsson, H.: A Dolbeault-Grothendieck lemma on a complex space via Koppelman formulas. Invent. Math. 190, 261-297 (2012)

7. Andersson, M., Wulcan, E.: Residue currents with prescribed annihilator ideals. Ann. Sci. École Norm. Super. 40, 985-1007 (2007)

8. Andersson, M.: A global Brianọ-Skoda-Huneke-Sznajdman theorem. Math. Scand. 122, 31-52 (2018)

9. Andersson, M., Lärkäng, R.: The $\bar{\partial}$-equation on a non-reduced analytic space. Math. Ann. arXiv: 1703.01861

10. Andersson, M., Wulcan, E.: Direct images of semi-meromorphic currents. Ann. Inst. Fourier 68, 875900 (2018)

11. Eisenbud, D.: Commutative Algebra. With a View Toward Algebraic Geometry. Graduate Texts in Mathematics, vol. 160. Springer, New York (1995)

12. Eisenbud, D.: The Geometry of Syzygies. A Second Course in Commutative Algebra and Algebraic Geometry. Graduate Texts in Mathematics, vol. 229. Springer, New York (2005)

13. Eisenbud, D., Goto, S.: Linear free resolutions and minimal multiplicity. J. Algebr. 88, 89-133 (1984)

14. Fornaess, J.E., Gavosto, E.A.: The Cauchy Riemann equation on singular spaces. Duke Math. J. 93(3), 453-477 (1998)

15. Fornaess, J.-E., Øvrelid, N., Vassiliadou, S.: Local $L^{2}$ results for $\bar{\partial}$ : the isolated singularities case. Intern. J. Math. 16, 387-418 (2005)

16. Fornaess, J.-E., Øvrelid, N., Vassiliadou, S.: Semiglobal results for $\bar{\partial}$ on a complex space with arbitrary singularities. Proc. Am. Math. Soc. 133, 2377-2386 (2005)

17. Götmark, E.: Weighted integral formulas on manifolds. Ark. Mat. 46, 43-68 (2008)

18. Götmark, G., Samuelsson, H., Seppänen, H.: Koppelman formulas on Grassmannians. J. Reine Angew. Math. 640, 101-115 (2010)

19. Helgesson, P.: Cauchy-Green formulas on a Riemann surface, Master thesis, Gothenburg (2010)

20. Henkin, G.: Cauchy-Pompeiu type Formulas for d-Bar on Affine Algebraic Riemann Surfaces and Some Applications. Geometry, and Topology. Springer, New York, Perspectives in Analysis (2012)

21. Henkin, G., Polyakov, P.: The Grothendieck-Dolbeault lemma for complete intersections. C. R. Acad. Sci. Paris Ser. I Math. 308(13), 405-409 (1989)

22. Henkin, G., Polyakov, P.: Residual d-bar-cohomology and the complex Radon transform on subvarieties of CPn. Math. Ann. 354, 497-527 (2012)

23. Henkin, G., Polyakov, P.: Explicit Hodge-type decomposition on projective complete intersections. J. Geom. Anal. 26, 672-713 (2016)

24. Henkin, G., Polyakov, P.: Explicit Hodge decomposition on Riemann surfaces. Math. Z. 1-18 (2017)

25. Lärkäng, R., Ruppenthal, J.: Koppelman formulas on the A_1-singularity. J. Math. Anal. Appl. 437, 214-240 (2016)

26. Lärkäng, R., Ruppenthal, J.: Koppelman formulas on affine cones over smooth projective complete intersections. Indiana Univ. Math. J. arXiv:1509.00987

27. Malgrange, B.: Sur les fonctions différentiables et les ensembles analytiques. Bull. Soc. Math. Fr. 91, 113-127 (1963) 
28. Øvrelid, N., Ruppenthal, J.: $L^{2}$-properties of the $\bar{\partial}$ and the $\bar{\partial}$-Neumann operator on spaces with isolated singularities. Math. Ann. 359, 803-838 (2014)

29. Øvrelid, N., Vassiliadou, S.: Solving $\bar{\partial}$ on product singularities. Complex Var. Elliptic Equ. 51, 225-237 (2006)

30. Øvrelid, N., Vassiliadou, S.: $L^{2}-\bar{\partial}$-cohomology groups of some singular complex spaces. Invent. Math. 192(2), 413-458 (2013)

31. Pardon, W.: The $L^{2}-\bar{\partial}$-cohomology of an algebraic surface. Topology 28(2), 171-195 (1989)

32. Pardon, W., Stern, M.: $L^{2}-\bar{\partial}-$ cohomology of complex projective varieties. J. Am. Math. Soc. 4(3), 603-621 (1991)

33. Pardon, W., Stern, M.: Pure Hodge structure on the $L \_2$-cohomology of varieties with isolated singularities. J. Reine Angew. Math. 533, 55-80 (2001)

34. Ruppenthal, J.: The $\bar{\partial}$-equation on homogeneous varieties with an isolated singularity. Math. Z. 263, 447-472 (2009)

35. Ruppenthal, J.: $L^{2}$-theory for the $\bar{\partial}$-operator on compact complex spaces. Duke Math. J. 163, 28872934 (2014)

36. Ruppenthal, J., Zeron, E.: An explicit $\bar{\partial}$-integration formula for weighted homogeneous varieties II. Forms of higher degree. Mich. Math. J. 59, 283-295 (2010)

37. Ruppenthal, J., Zeron, S.: An explicit $\bar{\partial}$-integration formula for weighted homogeneous varieties (2007). arXiv:0803.0136 